\title{
A Study on the Use of Cephalosporins in Patients with Diabetic Foot Infections
}

\author{
Prathap Balakrishna ${ }^{1}$, Dharati Shah ${ }^{2}$, Githa Kishore ${ }^{2}$, Sneha Keerthi ${ }^{2}$ \\ ${ }^{1}$ Kempegowda Institute of Medical Sciences Bengaluru, India. \\ ${ }^{2}$ Visveswarapura Institute of Pharmaceutical Sciences, Banashankari $2^{\text {nd }}$ stage, Benagaluru-560070, India.
}

\begin{abstract}
Diabetic foot problems such as ulcerations, infections and gangrene are dreadful complications of diabetes mellitus and a major cause of hospitalization. Overall $15 \%$ of individuals with diabetes mellitus will have foot ulcers during their lifetime. Diabetic foot infected patients are usually prescribed with broad spectrum antibiotics. Our study was done to evaluate the sensitivity pattern of cephalosporins in diabetic foot patients and to assess their prescribing patterns. We carried out a prospective study in the surgery department of a tertiary care hospital on 77 patients who had diabetic foot infections. Prescribing patterns of cephalosporins and wound culture and sensitivity testing on these patients was studied for a period of 6 months. Staphylococcus aureus was the most common gram positive pathogen isolated, E.coli, Klebsiella, Pseudomonas and Citrobacter were the common gram negative organisms isolated. Ceftriaxone was the most commonly prescribed antibiotic. Sensitivity pattern showed that $41.5 \%$ of gram positive cocci and $25 \%$ of gram negative bacilli were sensitive to Ceftriaxone. Ceftriaxone in our study was found to be an effective drug provided appropriate barrier techniques were used to prevent nosocomial spread. Organisms which were originally sensitive developed resistance approximately in a span of two weeks, probably due to antibiotic pressure. Hence there should be an active infection control team which can monitor the prevalent organisms and their antibiograms and periodically inform the clinicians. The pharmacists should also be included in the team, apart from the microbiologist, the surgeon and the nursing staff.
\end{abstract}

Key words: Cephalosporins, Diabetes, Diabetic foot infections, Prescribing patterns, Sensitivity pattern.

\section{INTRODUCTION}

Diabetes, considered as a disease of developed countries, is one of the endocrine disorders that have reached epidemic proportions worldwide. ${ }^{1}$ There are currently 285 million people living with diabetes worldwide, and the number of affected people is predicted to reach 438 million by $2030,{ }^{2}$ with 62 million diabetic individuals currently diagnosed with the disease in India. ${ }^{3}$ It is predicted that by 2030 diabetes mellitus may afflict up to 79.4 million individuals in India as a consequence of longer life expectancy, sedentary lifestyle and changing dietary patterns. ${ }^{4}$

Complications associated with diabetes arise chiefly from the disruption of the vascular system which can result in inadequate cir- culation to the peripheral body. This places the foot at higher risk of ulceration and infection. ${ }^{1}$

Diabetic foot ulcer is a major complication of diabetes mellitus, and probably the major component of the diabetic foot. A series of multiple mechanisms, including decreased cell and growth factor response, leads to diminished peripheral blood flow and decreased local angiogenesis, all of which can contribute to lack of healing in persons with diabetic foot ulcers. The foot ulcer is a leading cause of hospital admissions for people with diabetes in the world and is a major morbidity associated with diabetes, often leading to pain, suffering, and a poor quality of life for patients. ${ }^{5}$ Overall $15 \%$ of
DOI: $10.5530 /$ ijopp.7.4.6

Address for correspondence: Dr. GithaKishore Professor \& Head Department of Pharmacy Practice Visveswarapura Institute of Pharmaceutical Sciences, Bengaluru. Banashankari $2^{\text {nd }}$ stage, Benagaluru-560070.

Phone:9886025699

Email:ngkishore@gmail.com

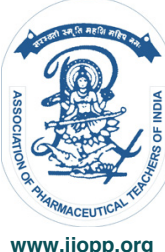


individuals with diabetes mellitus will have foot ulcer during their lifetime and in most developed countries, the annual incidence of foot ulceration amongst people with diabetes is about $2 \% .{ }^{6,7} \mathrm{In}$ these countries, diabetes is the most common cause of non-traumatic amputation; approximately $1 \%$ of people with diabetes suffer a lower-limb amputation. ${ }^{7}$

$85 \%$ of diabetes-related lower extremity amputations are preceded by a foot ulcer. In developed countries one in every six people with diabetes will have an ulcer during their lifetime. ${ }^{8}$

Diabetes and foot problems are almost synchronous. Pseudomonas spp., Enterococcus spp. and Proteus spp. are responsible for extensive tissue destruction with the poor blood circulation to the foot. ${ }^{9}$ Today antimicrobials remain the first line therapy for conquering bacterial infections. Treatment with these drugs is considered as a two-edged sword. As antimicrobial agents have been misused or overused, bacteria have fought back with a selection process by which certain strains are no longer susceptible to one or more agents. Each new use of these drugs, in fact, contributes to evolution of resistant microorganisms. As a result bacteria that once seemed to be losing the battle for survival have reemerged to create therapeutic dilemmas with resulting increased risk of treatment failure and disease complications. ${ }^{10}$

The cephalosporin antibiotics have become a major part of antibiotic formulary for hospitals in affluent countries. Clinicians choose empirical therapy with a cephalosporin whilst awaiting microbiological and other tests, since bacterial identification and antimicrobial testing usually requires $24-48 \mathrm{hr}$. The broad spectrum capability of these drugs, however, encourages rapid overgrowth of some microorganisms that are neither eliminated nor inhibited by therapy, as cephalosporins are not active against all the bacteria. These organisms not only have pathogenic potential, they may also be multiply resistant to antibiotics. There is evidence that cephalosporin usage is the most important factor in the selection and propagation of microorganisms such as Clostridium difficile, methicillin resistant Staphylococcus aureus, Penicillin-resistant Pneumococci, multiply resistant coliforms and vancomycinresistant Enterococci, the continuing increase of which threatens the future of antimicrobial therapy. ${ }^{11}$

Strict adherence and well accepted guidelines, along with caution in use of broad-spectrum antimicrobial agents, represents the best strategy for preventing the emergence and spread of nosocomial multidrug-resistant pathogens. ${ }^{12}$

Foot infections, which account for more hospital admissions than any of the other long-term complications of diabetes, are associated with increasing morbidity and mortality in diabetic patients. However, nosocomial pathogens are playing an important role in increasing morbidity and mortality of diabetic foot infections. ${ }^{13}$ Foot problems account for up to $15 \%$ of healthcare resources in developed countries and $40 \%$ in developing countries and anti-microbial resistance in the hospital setting has emerged as an important variable influencing patient outcomes and overall resource utilization. ${ }^{8}$

Prescribing practices of surgeons has become a major factor in today's scenario for treating foot infections. Inappropriate use of cephalosporins has become a major problem in the treatment, as there has been marked increase in resistivity seen among bacterial pathogens due to over prescribing or under prescribing of antibiotics.

Hence, an attempt was made here to study the prescribing patterns of cephalosporins by surgeons in the treatment of diabetic foot infections with a study on sensitivity testing and effect of nosocomial pathogens in a hospital set up.

\section{MATERIALS AND METHODS}

We performed a prospective 6 months study on 77 patients to know the prescribing, as well as the sensitivity patterns of Cephalosporins in patients with diabetic foot ulcers.

This study was conducted in the inpatient Surgery Department of, a tertiary care hospital which provides specialized health care services to all strata of people in and around Bengaluru.

The entire project was conducted according to the guidelines set by the Ethical Committee of the hospital.

All the patients with Type 1 and Type 2 Diabetes between the ages of $40-80$ years, admitted to the surgical department with a diabetic foot infection during the study period were included in the study. Diabetic foot infected patients prescribed with treatment other than Cephalosporins were excluded from the study.

A total of 77 patients who met the study criteria, diagnosed with diabetic foot, gangrene and cellulitis were observed for the study and were identified from the surgical inpatient wards. Treatment charts of the patients were reviewed prospectively for the prescription patterns of cephalosporins by the clinicians and were followed up with culture and sensitivity reports.

Data collected from each of the patients, included their demographic details, drug prescribed empirically, after first culture and subsequent repeat cultures and sensitivity reports.

All the above data was recorded in a data collection form for further analysis and interpretation. The data 


\begin{tabular}{|c|c|c|c|c|c|}
\hline $\begin{array}{l}\text { Drug and combination of } \\
\text { drugs }\end{array}$ & Dosage & $\begin{array}{l}\text { Empirical } \\
\mathrm{N}=77(\%)\end{array}$ & $\begin{array}{l}\text { After first culture } \\
n=77(\%)\end{array}$ & $\begin{array}{l}\text { After repeat } \\
\text { culture one } \\
n=48(\%)\end{array}$ & $\begin{array}{l}\text { After repeat } \\
\text { culture two } n=9(\%)\end{array}$ \\
\hline $\begin{array}{l}\text { Ceftriaxone } \\
\text { (Ci) }\end{array}$ & $1 \mathrm{gm} \mathrm{IV} \mathrm{bd}$ & $41(53.24)$ & $26(33.76)$ & $5(10.41)$ & $1(11.1)$ \\
\hline $\begin{array}{l}\text { Cefotaxim } \\
\text { (Ce) }\end{array}$ & $1 \mathrm{gm} \mathrm{IV} \mathrm{bd}$ & $4(5.19)$ & $13(16.38)$ & $6(12.50)$ & $1(11.11)$ \\
\hline $\begin{array}{l}\text { Cefoperazone+Salbactum } \\
\text { (Cfs) }\end{array}$ & $1.5 \mathrm{gm} \mathrm{IV} \mathrm{bd}$ & $28(36 . .36)$ & 19 (24.67) & $10(20.38)$ & $4(44.44)$ \\
\hline $\begin{array}{l}\text { Cefepime } \\
(\mathrm{Cpm})\end{array}$ & $1 \mathrm{gm} \mathrm{IV} \mathrm{bd}$ & $3(3.89)$ & $3(3.89)$ & $1(2.08)$ & - \\
\hline $\begin{array}{l}\text { Cefixime } \\
\text { (Cfx) }\end{array}$ & $1 \mathrm{gm} \mathrm{IV} \mathrm{bd}$ & $1(1.29)$ & $2(2.59)$ & $1(2.08)$ & - \\
\hline $\begin{array}{l}\text { Drugs other than } \\
\text { cephalosporins }\end{array}$ & - & - & $14(18.18)$ & $25(52.08)$ & $3(33.33)$ \\
\hline
\end{tabular}

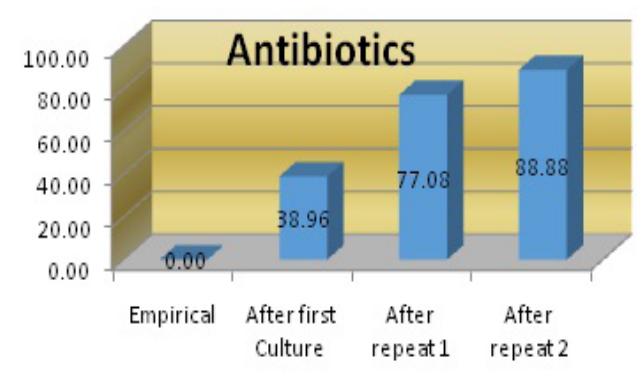

Figure 1: Bar diagrams showing percentage of drugs prescribed other than Cephalosporins

The prescribing pattern of drugs other than cephalosporins after each sensitivity testing. It clearly shows that after each sensitivity testing, the percentage of drugs prescribed other than cephalosporins had increased

of the first culture and sensitivity reports of 77 patients, repeat one-culture sensitivity reports of 48 patients and repeat two culture and sensitivity reports of 9 patients were collected. First culture and sensitivity reports were collected to determine the effect of empirical treatment. Repeat culture and sensitivity reports were collected to determine the effect of the cephalosporins and presence of nosocomial pathogens.

Treatment failure was defined as any change in the antibiotic prescription given due to the presence of different organisms with differences in sensitivity on repeat culture performed. If the patient's ulcer was not healing, repeat procedures like debridement, amputation etc were one of the options considered.

Cross-tabulation for SPSS (Statistical Package for Social Sciences) Software has been used to find the prescription pattern with organism isolated each time. Significance was reported by 95\% CI (Confidence Interval) which was calculated by binomial probability method.

\section{RESULT}

Table 1 shows that most commonly prescribed cephalosporins empirically were ceftriaxone in 41 (53.24\%) Indian Journal of Pharmacy Practice, Vol 7, Issue 4, Oct-Dec, 2014
Figure 2: Pie chart showing different types of diabetic foot infection

Among 77 patients, 40 (51.95\%) patients were diagnosed with diabetic foot ulcer followed by $21(27.27 \%)$ cellulitis and $16(20.78 \%)$ gangrene

patients followed by cefoperazone + salbactum in 28 $(36.36 \%)$ patients. Table 1 shows that, after first culture ceftriaxone was given to $26(33.76 \%)$ patients and cefoperazone + salbactum to $19(24.67 \%)$ patients. After the antibiograms, percentage of drugs other than cephalosporins prescribed were increased (Figure 1). After first culture other antibiotics prescribed were 14 (18.18\%) followed by in repeat one $25(52.08 \%)$ and in repeat two $3(33.33 \%)$.

Out of 307 antibiograms of the patients, 195 (63.51\%) showed growth; of which $41(21.02 \%)$ yielded grampositive cocci followed 128 (65.64\%) gram-negative bacilli (enterobacteriaceae $95(48.71 \%)$ and $26(13.3 \%)$ polymicrobials. Gram-positive cocci isolated were enterococcus $23(56 \%)$ followed by coagulase negative Staphlococcusaureus 12 (29.3\%). Gram-negative bacilli isolated were E.coli $38(29.7 \%)$ followed by Klebsiella $28(21.9 \%)$.

Polymicrobials were combinations of:

Gram-negative bacilli and Gram-negative bacilli

Gram-positive cocci and Gram-negative bacilli

Gram-positive cocci and Gram-positive cocci. 


\begin{tabular}{ll}
\hline Table 2: Patients' diabetic history on admission \\
\hline Type 2 diabetes & $75(97.40 \%)$ \\
\hline Type 1 diabetes & $2(2.59 \%)$ \\
Diagnosed previously & $69(89.61 \%)$ \\
Diagnosed on admission & $8(10.38 \%)$ \\
Duration of diabetes & 3 months to 20 years \\
On OHA & $57(82.6 \%)$ \\
On Insulin & $12(17.4 \%)$ \\
RBS on admission & $175-325(\mathrm{mg} / \mathrm{dl})$ \\
Surgical Intervention & - \\
Debridement & $14(18.18 \%)$ \\
Toe disarticulation & $7(9.09 \%)$
\end{tabular}

OHA - Oral hypoglycemic agents : RBS - Random blood sugar

Out of 77 patients, 75 (97.40\%) patients had type 2 diabetes and 2 (2.59\%) has type 1 diabetes.

From the Figure 3, E.coli was the most prevalent organism in diabetic foot infection in this hospital.

Out of 77, $30(38.96 \%)$ patients managed with antibiotics alone, while $47(61.04 \%)$ patients underwent surgical intervention with concomitant antibiotics. Debridement was the most frequent surgical intervention in 26 $(33.76 \%)$ patients followed by toe disarticulation in 14 $(18.18 \%)$ and amputation in $7(9.09 \%)$ patients.

\section{DISCUSSION}

A total of 77 patients who fulfilled the inclusion and exclusion criteria and who were admitted to our study center were included in this study, which was carried out for a period of six months.

Among the 77 patients with diabetic foot infections from surgical units, $70 \%$ were male and $30 \%$ were female. According to Llanes, male population accounts for more hospital admission than females in diabetic foot infections. ${ }^{14}$ In our study $90.4 \%$ patients had type 2 diabetes and only $9.6 \%$ had type 1 diabetes (Table 2 ). We observed that $48.2 \%$ patients had ulcers followed by $27.27 \%$ with cellulitis and $20.78 \%$ with gangrene (Figure

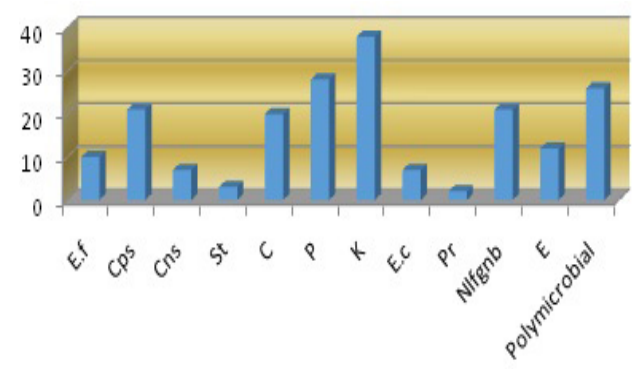

Figure 3: Bar diagram showing frequency distribution of organisms

Where, E.f- Enterococcus; Cps- Coagulase positive Staphylococcus aureus; CnsCoagulase negative Staphylococcus aureus; St- Streptococcus; C- Citrobacter; P- Psuedomonas; K- Klebsiella; E.c- E.coli; Pr- Proteus; Nlfgnb- Non-lactose fermentative gram-negative bacilli other than Pseudomonas; E- Enterobacter)
2). According to a similar study by Anandi $C$ on patients with diabetic foot lesions, $56 \%$ had ulcer followed by $26.1 \%$ with cellulitis and $15.9 \%$ with gangrene. ${ }^{15}$

The present study illustrated that the infection was due to gram-positive cocci, gram-negative bacilli and polymicrobials. Among these $65.6 \%$ gram-negative bacilli (enterobacteriaceae group 48.71\%) followed by $21.02 \%$ gram-positive cocci and $14.87 \%$ polymicrobials were isolated. Out study revealed that E.coli (19.5\%), Klebsiella (14.35\%), Pseudomonas (10.8\%) and Citrobacter $(10.3 \%)$ were the most common gram-negative organisms isolated. Staphylococcus aureus (14.4\%) and Enterococcus $(11.8 \%)$ were the most common grampositive pathogen isolated in our study.

These results were comparable with a similar study which was conducted in Bangalore by Vidya $\mathrm{D}$ et al, in diabetic foot infections where the most commonly isolated organisms were Staphylococcus aureus, Citrobacterspp, Pseudomonas spp, Enterococcus and Pneumococci. ${ }^{16}$

According to Motta RN et al, enterobacteriaceae group $(97.8 \%)$ were the most frequently isolated bacteria. ${ }^{17}$ According to David Burgess S, Extended-Spectrum $\beta$-Lactamase (ESBL)-producing organisms are an increasing problem for practitioners dealing with infectious disease. Escherichia coli, Klebsiellapneumoniae and Klebsiellaoxytoca are the most common ESBLproducing pathogens. ${ }^{18}$

Sensitivity testing was done for patients with gram-negative bacilli in diabetic foot infection at our study center and it was found that organisms showed increased resistance patterns to cephalosporins, while in case of infections due to gram-positive cocci, cephalosporins on them was not studied separately.

According to the reporting at Inter science Conference on Antimicrobial Agents and Chemotherapy (ICAAC) conference, Extended Spectrum $\beta$-Lactamase (ESBLs) are $\beta$-lactamases that hydrolyze extended-spectrum cephalosporins with an oxyimino side chain. These cephalosporins include cefotaxim, ceftriaxone and ceftazidime, as well as the oxyimino-monobactamaztreonam. The clinical relevance of ESBLs has been well documented by numerous published case reports describing clinical failures with the use of third generation cephalosporins such as these oxyimino-cephalosporins (cefotaxime, ceftriaxone and ceftazidime) as well as with the use of cefoxetin and the fourth generation cephalosporin, cefepime. Thus, the problem of ESBLs is clinically important, yet remains relatively unappreciated by most clinicians. This is because many clinical microbiology laboratories continue to mistakenly report these gram-negative bacillary isolates as susceptible due to difficulties in identifying those isolates which possess this important beta-lactamases. ${ }^{19}$

Indian Journal of Pharmacy Practice, Vol 7, Issue 4, Oct-Dec, 2014 
In the present study, injection ceftriaxone $(53.24 \%)$ was the most commonly prescribed drug followed by injection cefoperazone + salbactum $(36.36 \%)$ and injection cefotaxime $(5.19 \%)$.

Out of 77 patients, $41(53.24 \%)$ patients were prescribed with ceftriaxone as empirical treatment and pus samples were sent for culture and sensitivity test. In 19 $(46.3 \%)$ patients, there were either no growth or normal flora and $22(53.7 \%)$ patient's yielded growth. Antibiograms were available for only 17 isolates. Out of these 17, 8 gram-negative bacilli (47.1\%) and 1 polymicrobial $(5.9 \%)$ were resistant to empirical drug after first culture [With 95\% CI of $26.17-69.04$ and 95\% CI of $1.05-$ 26.98 respectively].

Without following the antibiograms, ceftriaxone was discontinued in 2 (11.8\%) (95\% CI: 3.29-34.34) patients of the 17 isolates, even though it was sensitive and in 5 $(29.4 \%)$ (95\% CI: 13.28 -53.13) patients where antibiograms were not available. It is prudent to inform the microbiologist to include the empirical drug for testing sensitivity pattern of organism in antibiogram, before continuing or discontinuing the drug.

In $4(23.5 \%)(95 \%$ CI: $9.56-47.26)$ patients (3 gramnegative and 1 gram-positive organisms isolated), the patients were sensitive to organisms after first culture and developed resistance after repeat culture. Irrational withdrawing or long-term prescription of cephalosporins can lead to such resistance.

In 22 patients, repeat culture was done. Of which $18.2 \%$ yielded either commensals or remained sterile. Of the remaining where there was growth 13 (59.1\%) (95\% CI: 49.13-87.50) patients showed different organisms. Predominant organisms isolated in the repeat culture were: Pseudomonas, Klebsiella, Citrobacter, E.coli, Streptococcus and Enterococcus. The patients could have acquired these organisms as nosocomial spread. Out of 13 different organisms isolated in the repeat culture, 10 (76.92\%, 95\% CI (49.7\%-91.8\%) organisms were resistant to the drug.

Ceftriaxone is an effective drug, provided appropriate barrier techniques are used in preventing nosocomial spread. Organisms which were originally sensitive developed resistance approximately in span of two weeks which can be probably due to antibiotic pressure.

\begin{tabular}{lc}
\hline Table 3: Management & \\
\hline MANAGEMENT & No. of cases \\
\hline Antibiotic alone & $30(38.96 \%)$ \\
Surgical intervention & $47(61.04 \%)$ \\
* Debridement & $26(33.76 \%)$ \\
* Toe disarticulation & $14(18.18 \%)$ \\
* Amputation & $7(9.09 \%)$ \\
\hline
\end{tabular}

Indian Journal of Pharmacy Practice, Vol 7, Issue 4, Oct-Dec, 2014
Out of 77 patients, $28(36.36 \%)$ patients were prescribed with cefoperazone + salbactum empirically. After treatment, pus samples were sent for culture and sensitivity. In $6(21.4 \%)$ patients there was no growth or normal flora. Out of the remaining, $22(78.6 \%)$ patients yielded growth, antibiograms were available for 20 (71.4\%) patients.

Out of 20 patients, 8 (40\%) (95\% CI: 21.88-61.34) gramnegative organisms' isolates, $3(15 \%)$ gram-positive and $1(5 \%)$ polymicrobial were resistant to first culture [12 (60\%), 95\% CI: 38.06-78.12].

In 5 (25\%) (95\% CI: 11.19-46.87) patients, gram-negative organisms isolated were sensitive after the first culture and developed resistance in the repeat culture. Biostatistically a negative significance was observed with respect to the combination of cefoperazone + salbactum. This shows that cefoperazone + salbactum may not be an ideal alternative for ceftriaxone in these patients.

In $22(78.6 \%)$ patients, repeat culture was done, out of which $4(18.1 \%)$ yielded either commensals or remained sterile. Of the remaining 18 where there was growth, in 11 (61.11\%) (95\% CI: 38.62-79.69) patients different organisms were isolated. Out of 11 different organisms isolated, 5 (45.45\%) (95\% CI: 21.3-71.9) were resistant to empirical drug.

According to Marin KH, gram-positive bacterial infections are becoming more prevalent within the hospital setting. Among gram-positive bacteria, methicillin-resistant S.aureus (MRSA) and vancomycin-resistant Enterococci (VRE) seem to be the most problematic in terms of their occurrence and impact on the clinical outcomes of hospitalized patients. Similarly, increasing resistance to penicillin and cephalosporins has become an important issue for one of the most prevalent causes of community-acquired-gram-positive infection. ${ }^{13}$

According to Mitchell SJ, resistance to third-generation cephalosporins in gram-negative nosocomial pathogen is a formidable problem, associated with adverse clinical outcomes and increased hospital costs. ${ }^{20}$

According to Rahal JJ, extensive cephalosporin class restriction significantly reduced nosocomial, plasmidmediated, cephalosporin-resistant Klebsiella infection and colonization. ${ }^{21}$

Nosocomial infection and surgical intervention like debridement, toe disarticulation and amputation probably were the factors contributing to treatment failure. Antibiotic pressure (prolonged use of antibiotics) may (Table 3) also contribute to treatment failure.

Patients have to be counseled regarding care to be taken to prevent foot infections, as diabetic patients are vulnerable to foot infections. 


\section{CONCLUSION}

In our study, we found that, gram-negative bacilli showed increased resistance pattern to cephalosporins, while in case of infections due to gram-positive cocci, cephalosporins were effective.

Ceftriaxone is an effective drug provided appropriate barrier techniques are implemented in preventing nosocomial spread. Organisms which were originally sensitive, developed resistance approximately in a span of two weeks, which can probably be due to antibiotic pressure. Treatment for longer duration may result in drug resistance.

Hence treatment failure can probably be due to nosocomial infection and antibiotic pressure. Hence, there should be an active infection control team, which can monitor the prevalent organisms and their antibio- grams, and periodically inform the clinicians. The pharmacist should also be included in the team apart from microbiologist, surgeon and nursing staff.

\section{CONFLICT OF INTEREST}

Authors declared no conflict of interest

\section{ACKNOWLEDGMENT}

We are thankful to the principal and the staff of VIPS and KIMS for permitting us to carry out the study, collect data and for all the cooperation extended while carrying out the study.

Funding- no funding sources

Ethical approval- The study was approved by the institutional ethics committee.

\section{REFERENCES}

1. Barbara Currie, Ehud Ur and Thomas Ransom. Clinical Practice Guidelines: A Global Perspective. In: (eds.)The Epidemiology of Diabetes Mellitus. 2nd ed. UK: Wiley; 2008. p641.

2. Frank B, Hu. Globalization of Diabetes: The role of diet, lifestyle, and genes. Diabetics Care. June 2011; 34(6): 1247-57.

3. Seema, Abhijeet, Kaveeshwar, Jon Cornwall. The current state of diabetes mellitus in India. Australasian Medical Journal 2014; 7(1): 45-8.

4. Wild S, Roglic G, Green A, Sicree R, King H. Global prevalence of diabetesestimates for the year 2000 and projection-s for 2030. Diabetes Care. 2004; 27(3): 1047-53.

5. Brem H, Tomic-Canic M. Cellular and molecular basis of wound healing in diabetes. Journal of Clinical Investigation 2007; 117(5): 1219-22.

6. Vileikyte L. Diabetic foot ulcers: a quality of life issue. Diabetes Metab Res Rev. 2001 17(4): 246-9.

7. Andrew Boulton. The diabetic foot: epidemiology, risk factors and the status of care. Diabetes voice.2005;50(Special Issue):5-7.

8. International Diabetes Federation. The Global Burden. IDF Diabetes Atlas Fifth Edition; 2012

9. Suresh A, Muthu G, Srivani R, Moses A. Aerobic bacterial resistance in diabetic foot ulcer from Chennai. International Journal of Pharma and Bio Sciences 2011; 2(2): 517-28.

10. Hooton TM, Levy SB. Confronting the antibiotic resistance crisis: making appropriate therapeutic decisions in community medical practice. Infect Med. 2004; 21(3): 114-22.

11. Dancer SJ. The problem with cephalosporins. Journal of Anti-microbial Chemotherapy $2001 ; 48(4)$ : 463-78.

12. Patterson JE. Antibiotic utilization is there an effect on anti-microbial chemotherapy $2001 ; 118(2):$ 4265-305.

13. Kollef MH. Antibiotic resistance among gram-positive bacteria in the hospital setting: what can we do about it? www.medscape.comlviewprograml623 author access May 20, 2006.

14. Llanes LRI, Pena AC, Cauton-Valera R. Clinical, microbiological profile and outcome of diabetic patients with foot ulcers admitted al Quirino Memorial Medical Centre. Phil J Microbial Infect Dis. 2001; 30(3): 101-7.

15. Anandi C, Alayuraja D, Natarajan V, Ramanathan M, Subramaniam CS, Thulasiram $\mathrm{M}$, et al. Bacteriology of diabetic foot lesions. Indian $\mathrm{J}$ Med Microbial. 2004; 22(3): 175-8.

16. Vijaya D, Lakshmikanth, Shesshadri. Bacteriology of diabetic foot infection. Biomedicine 2000; 20(3): 176-9.

17. Motta RN, Oliveria MM, Magalhaes PSF, Dias AM, Aragao LP, Forti AG. Plasmid-mediated extended-spectrum $\beta$-lactamase-producing strains of enterobacteriaceae isolated from diabetic foot infections in a Brazilian diabetic center. Braz J Infect Dis 2003 Apr; 7 (2): 129-34.

18. Burgess DS, Hall RG, Lewis JS, Jorgensen JH, Patterson JE. Clinical and microbiologic analysis of a hospital's extended spectrum $\beta$-lactamase producing isolates over a two-year period. www.medscape.com March 29, 2006.

19. Stratton C. Extended-spectrum $\beta$-lactamases: An unappreciated global problem. $42^{\text {nd } I n t e r s c i e n c e ~ C o n f e r e n c e ~ o n ~ A n t i-m i c r o b i a l ~ a g e n t s ~ a n d ~ c h e m o t h e r a p y . ~}$ www.medscape.com/viewprogram/2003-authors June 29, 2006.

20. Schwaber MJ, Cosgrove SE, Gold HS, Kaye KS, Carmeli Y. Fluoroquinolones protective against cephalosporin resistance in gram-negative nosocomial pathogens. Emerg Infect Dis. 2004; 10(1): 94.

21. Rahal JJ, Uraban C, Horn D, Freeman K, Segal-Maurer S, Maurer J, et al. Class restriction of cephalosporin use to control total cephalosporin resistance in nosocomial Klebsiella. JAMA. 1998; 280(14): 1233-7. 